\title{
Chemotherapy options versus "novel” therapies: how should we treat patients with malignant pleural mesothelioma
}

\author{
Maria J. Disselhorst, Paul Baas \\ Department of Thoracic Oncology, The Netherlands Cancer Institute, Plesmanlaan 121, 1066CX Amsterdam, The Netherlands \\ Contributions: (I) Conception and design: All authors; (II) Administrative support: None; (III) Provision of study materials or patients: None; (IV) \\ Collection and assembly of data: All authors; (V) Data analysis and interpretation: All authors; (VI) Manuscript writing: All authors; (VII) Final \\ approval of manuscript: All authors. \\ Correspondence to: Paul Baas, MD, PhD. Department of Thoracic Oncology, The Netherlands Cancer Institute, Plesmanlaan 121, 1066CX \\ Amsterdam, The Netherlands. Email: p.baas@nki.nl.
}

\begin{abstract}
Today there are several options for the treatment of patients with malignant pleural mesothelioma (MPM). The therapeutic arsenal has expanded from only chemotherapy with or without surgery in selected cases to a variety of new compounds that target the malignant cell or its micro-environment. Immunotherapy has been the latest achievement and now single arm and randomized studies are being presented. A renewed interest has occurred in the combination of surgery, chemotherapy and radiation therapy. In this review we present the available data on previous and running studies and try to give a recommendation how to select the best patient for the most optimal therapy.
\end{abstract}

Keywords: Malignant pleural mesothelioma (MPM); systemic treatment; clinical trial; surgery

Submitted Dec 09, 2020. Accepted for publication Jan 16, 2020.

doi: $10.21037 /$ tlcr.2020.01.16

View this article at: http://dx.doi.org/10.21037/tlcr.2020.01.16

\section{Introduction}

Until the change of the century patients with malignant pleural mesothelioma (MPM) were treated with best supportive care (BSC) and offered mostly single agent chemotherapy as part of a study. The median overall survival of this group was 7-8 months and only a hand full of chemotherapeutic agents gave responses of $15-20 \%$. One of the most informative studies before 2000 was the MS-01 study from the UK. In this three-arm study, BSC was compared to vinorelbine and to mitomycin plus vinblastine plus cisplatin. No statistical benefit was observed but a slight survival benefit for the vinorelbine arm was noted (1). It was until 2003 that the study by Vogelzang showed a clear benefit from the combination of cisplatin plus pemetrexed versus cisplatin monotherapy. This raised the median overall survival (OS) from 10 to 13 months (2). The combination has been the standard now for over 15 years and is considered the backbone to which other combinations can be tested. The basic idea of the success of this combination is based on the low expression of Thymidilate Synthase (TS) in patients with MPM allowing the multitarget antifolate (pemetrexed) to inhibit the generation of nucleotides in the malignant cells. This, combined with the DNA disrupting effect of cisplatin during the cell division lead to an improved mOS and a response rate of $35 \%$ (3).

In this review we describe the literature and latest data and try to recommend the best possible treatment.

\section{Angiogenesis inhibitors}

For growth of MPM cells, the vasculature plays an important role. Examination of histologic specimen have indicated that a variety of vascular growth factors play a role. A high micro-vessel count is often seen in patients with active growth of the tumor and are correlated with a worse prognosis. Many receptors have been identified that are activated in patients with MPM, like VEGF-R1 to 4, PDGF and PGF (4-6). Vascular endothelial growth 
Table 1 Angiogenesis inhibitors

\begin{tabular}{|c|c|c|c|}
\hline Inhibitors & Mode of action & Dose & General outcome \\
\hline Bevacizumab (10-12) & VEGF & 15 mg/kg i.v. q 3 wks with CT & $\begin{array}{c}\text { mOS } 18.8 \text { vs. } 16.1 \text { HR } 0.77 \\
\text { (significant) }\end{array}$ \\
\hline Cederanib $(13,14)$ & VEGFR1-3; c-KIT; PDGFR $\beta$ & 45 mg daily & PR 9-10\% significant toxicity \\
\hline \multirow{2}{*}{ Nintedanib (16) } & & & PFS: HR 1.01 \\
\hline & & & OS: HR1.12 \\
\hline Sorafenib CALGB $30307(17,18)$ & RAS/RAF/MEK; VEGF; c-KIT & 400 mg twice daily & PR 6\% \\
\hline Thalidomide (21) & & & OS: HR 1.2 \\
\hline Vatalanib (22) & VEFG; PDGF; c-KIT & 1,250 mg daily after CT & PR 6\% \\
\hline
\end{tabular}

$\mathrm{CT}$, chemotherapy; HR, hazard ratio.

factor (VEGF) is expressed in MPM, can promote tumor angiogenesis, but also directly stimulate tumor growth $(7,8)$. These observations have led to a variety of studies using anti-angiogenic drugs as shown in Table 1. Not only new compounds like small molecules have been tested but also older angiogenic inhibiting drugs like thalidomide. The latter has been tested in a phase III maintenance setting, where patients who did not progress after 4-6 courses of platinum plus pemetrexed, were randomized to receive observation or thalidomide until progression. Although the drug was well tolerated, there was no sign of activity at all compared to observation alone (21). Many phase 2, non-randomized studies have been performed with small molecules directed against the VEGF receptors. Most of these compounds had shown activity in other tumors like kidney cancer. Unfortunately, most of the studies did not show consistent activity in patients with MPM and response rates of $6-12 \%$ were noted. The toxicities were often reason for dose reduction or even discontinuation of the therapy. To date no small molecule has been identified to be used on larger scale $(9,13-20,22)$.

A special note must be made for the addition of bevacizumab to the standard of care. In the MAPS study in France, patients were randomized to receive the standard of care with or without bevacizumab in a dose of $15 \mathrm{mg} / \mathrm{kg}$ i.v. every 3 weeks. The drug could be given as a maintenance after a maximum of 6 courses of chemotherapy were administered. Two interesting observations could be made in this study; (I) there was a significant mOS benefit for the patients receiving bevacizumab of 2.8 months; (II) the mOS in the control arm had increased to 15 months (10). The latter observation indicates that there may have been a better selection of patients since the SoC reported only a 12-13 months mOS. It remains unclear if this observation is related to the selection for patients fit to receive bevacizumab or that the natural history of the disease has changed in the last 10-15 years. Nowadays, the addition of bevacizumab has been registered as possible new standard of care in some countries.

\section{Maintenance therapies}

The use of maintenance therapy has attracted attention in different tumor types and has been tested in patients with MPM. In the first phase III study reported, thalidomide was tested in a dose of $200 \mathrm{mg}$ orally until progression. As stated above, no difference in median progression free survival (PFS) was noted. The mPFS was 3.5 months in both groups with a HR of 0.99 (21). Pemetrexed has been tested as a maintenance drug in a randomized phase II trial. The data 
Table 2 Single agent checkpoint inhibitors

\begin{tabular}{|c|c|c|c|c|c|c|}
\hline Author, trial & Checkpoint inhibitor & Patients (n) & ORR (\%) & $\operatorname{DCR}(\%)$ & $\begin{array}{c}\text { PFS } \\
\text { (months) }\end{array}$ & OS (months) \\
\hline Alley, Keynote028 Phase 1B (28) & Pembrolizumab & 25 & 20 & 72 & 5.4 & 18 \\
\hline Popat, Promise-meso Phase III (30) & Pembrolizumab vs. chemotherapy & 73 vs. 71 & 22 vs. 6 & & 2.5 vs. 3.4 & 10.7 vs. 11.7 \\
\hline Quispel, Nivomes Phase II (31) & Nivolumab & 34 & 26 & 47 & 2.6 & 11.8 \\
\hline Scherpereel, MAPS-2 Phase II (33) & Nivolumab & 62 & 17 & 43 & 4.0 & 11.9 \\
\hline Hassan, Javelin Phase 1B (34) & Avelumab & 53 & 9 & 47 & 4.1 & 10.7 \\
\hline Maio, Determine Phase III (35) & Tremelimumab vs. placebo & 382 vs. 189 & 4.5 vs. 1.1 & 27.7 vs. 21.7 & 2.8 vs. 2.7 & 7.7 vs. 7.3 \\
\hline
\end{tabular}

of this study were presented as poster during ASCO 2019. The study suffered from a very slow accrual and with only 49 patients entered, no difference were observed in both mPFS (3.4 vs. 3.0 months) and mOS (16.3 vs. 11.4 months $\mathrm{P}=0.67)$. The study was stopped for slow accrual (23).

Recently a randomized phase II has been reported during ESMO 2019 with interesting outcomes. In the maintenance setting, gemcitabine was administered in a dose of $1,250 \mathrm{mg} / \mathrm{m}^{2}$ weekly $\times 2$ every 3 weeks. This regimen was compared to BSC and patients could enroll when no signs of progression were noted after 4-6 courses of platinum-pemetrexed. The drug was well tolerated but a number of patients had dose reductions or change in interval due to toxicity. The primary endpoint was met with an improvement of mPFS of 3 months compared to BSC (3.2 vs. 6.2 months). The HR of $0.42(0.28-6.3)$ and a $\mathrm{P}<0.0001$ makes this an interesting observation. Eagerly, the mOS data are awaited (24).

\section{Epigenetic interference}

Another cell cycle regulatory pathway which attracted interest and is transcription pathway of DNA. In this process, histone deacetylase (HDAC) regulates the timely transcription of DNA by unfolding parts of DNA from the histones. Vorinostat is a HDAC inhibitor with a small molecular weight $(<264 \mathrm{~g} / \mathrm{mol})$ and leads to induction and accumulation of acetylated histones. This results in a reduction of proliferation of cells, especially tumor cells. This oral medication was tested in second- and third-line treatment in one of the largest phase III studies reported. Despite a positive indication of success in the interim analysis, the final results of
661 randomized patients did not show any difference in mPFS or mOS (30.7 vs. 27.1 weeks mOS) (25). It was concluded that single agent HDAC inhibition is not an effective strategy and should probably be combined with other targeted approaches (26).

A more recent development is the observation that the Polycomb Repressor Complex (PRC) is involved in the suppression of tumor suppressor genes in mesothelioma. It was demonstrated that the Enhancer of Zeste Homolog 2 (EZH2) is over-expressed in MPM, and the related PRC2 is a potential therapeutic target in this tumor. Further studies of TCGA confirmed an up-regulation of EZH2 in MPM cells (27). In order to inhibit the EZH2/PCR2 complex, a drug named tazemetostat has been tested. This compound has now been tested in a small series of 74 patients with MPM, but has not resulted in a full publication (NCT02860286).

\section{Single agent immune checkpoint inhibitors}

In the past several years multiple promising data on immune checkpoint inhibitors (ICI) have been reported in the second or later lines (summarized in Table 2). Single agent PD-1 ICI have consistent objective response rates of about $20 \%$ in mainly phase II trials (28,29,31-34). Single agent CTLA-4 checkpoint inhibitor tremelimumab however did not show any benefit compared to placebo (35).

At ESMO 2019 meeting the PROMISE-meso trial was presented. An ETOP initiated phase III trial with pembrolizumab versus chemotherapy (gemcitabine or vinorelbin) in further lines. Although a significant difference in ORR was seen $(22 \%$ versus $6 \%, \mathrm{P}=0.004)$, it did not 
Table 3 combination checkpoint inhibitors

\begin{tabular}{|c|c|c|c|c|c|c|}
\hline Author, trial & Checkpoint inhibitors & Patients (n) & ORR (\%) & DCR (\%) & PFS (months) & OS (months) \\
\hline Disselhorst, Initiate Phase II (36) & Nivolumab + ipilimumab & 34 & 38 & 68 & 6.2 & NR \\
\hline Scherpereel, MAPS-2 Phase II (33) & Nivolumab + ipilimumab & 63 & 24 & 50 & 5.6 & 15.9 \\
\hline
\end{tabular}

result in a difference in PFS or OS. The ORR of $22 \%$ is consistent with the earlier phase II trials. Treatment related adverse events of grade 3 or higher were experienced in more patients in the chemotherapy group (19\% versus 24\%) (30). Whether a small subgroup exists that does have a survival advantage for ICI over chemotherapy is not yet known, neither how to select patients that will have a response. In most of the above-mentioned trials, tumors with PD-L1 expression have a higher response-rate to ICI than tumors without PD-L1 expression. But this is not consistent, and also tumors without PD-L1 expression have responses.

\section{Combination of immune checkpoint inhibitors with chemotherapy}

In line with the positive effect of combining chemotherapy and an immune checkpoint inhibitor in NSCLC, different phase II and III trials are ongoing, with different combinations (NCT02899195, NCT02784171, NCT03762018).

\section{Combination of immune checkpoint inhibitors}

In the last 2 years, three separate phase II trials testing a combination of checkpoint inhibitors were published, one combining durvalumab plus tremelimumab (NIBITMESO-1) and two with nivolumab plus ipilimumab (MAPS2 and INITIATE) $(33,36,37)$. These are summarized in Table 3. Response rates between $25 \%$ and $38 \%$ were seen, which seem a bit higher than from single agent PD-1 inhibitors. Whether this will induce a survival benefit is now being tested in a first line phase III trial randomizing between standard chemotherapy and nivolumab plus ipilimumab (Checkmate 743; NCT02899299). Results are being expected next year. The combination of nivolumab plus ipilimumab is already included in the NCCN guidelines. In line with the single agent ICI, selecting patients for the treatment seems crucial; but a biomarker is not yet found.

\section{Dendritic cell immunotherapy}

In dendritic cell immunotherapy autologous monocytederived dendritic cells are pulsed with allogenic tumor lysate from five different mesothelioma cell lines and reintroduced into the patient by a vaccination. In the first phase 1 trial 9 patients were treated with this (Mesopher) vaccination, which resulted in a DCR of $100 \%$ (38). This led to a randomized phase II/III trial testing maintenance vaccination versus observation after effective first-line chemotherapy. This European study is currently including patients (NCT03610360).

\section{Mesothelin targeted therapy}

Mesothelin is a cell surface glycoprotein normally expressed on mesothelial cells, and highly expressed in different cancers, especially in epithelioid mesothelioma. Thereby it is an interesting target for therapy, and different approaches are used over the last two decades (Figure 1).

One of the approaches is as a chimeric high-affinity monoclonal antibody (amatuximab), potentially this reduces tumor growth by inhibiting mesothelin binding to the extracellular substrate and by antibody-dependent cellular cytotoxicity. But in a multicenter phase II study, amatuximab in combination with pemetrexed and cisplatin failed to show a difference in PFS over historical controls (39).

Another way to target mesothelin is with immunotoxins. An antibody fragment that targets mesothelin is fused to a bacterial exotoxin payload, and after binding it is internalized by the cell via endocytosis and can induce apoptosis. Two different drugs have been, or are now being tested in clinical trials, SS1P and LMB-100. In SS1P a fragment of Pseudomonas exotoxin A (PE38) is fused. As single agent it has modest efficacy, and problem is induction of rapidly evolving antibodies which neutralize the drug (40). The newer drug LMB-100 has a designed PE (PE24) and is designed to be less immunogenic and thereby less toxic; and is now being tested in clinical trials (NCT03644550, NCT02798536). 


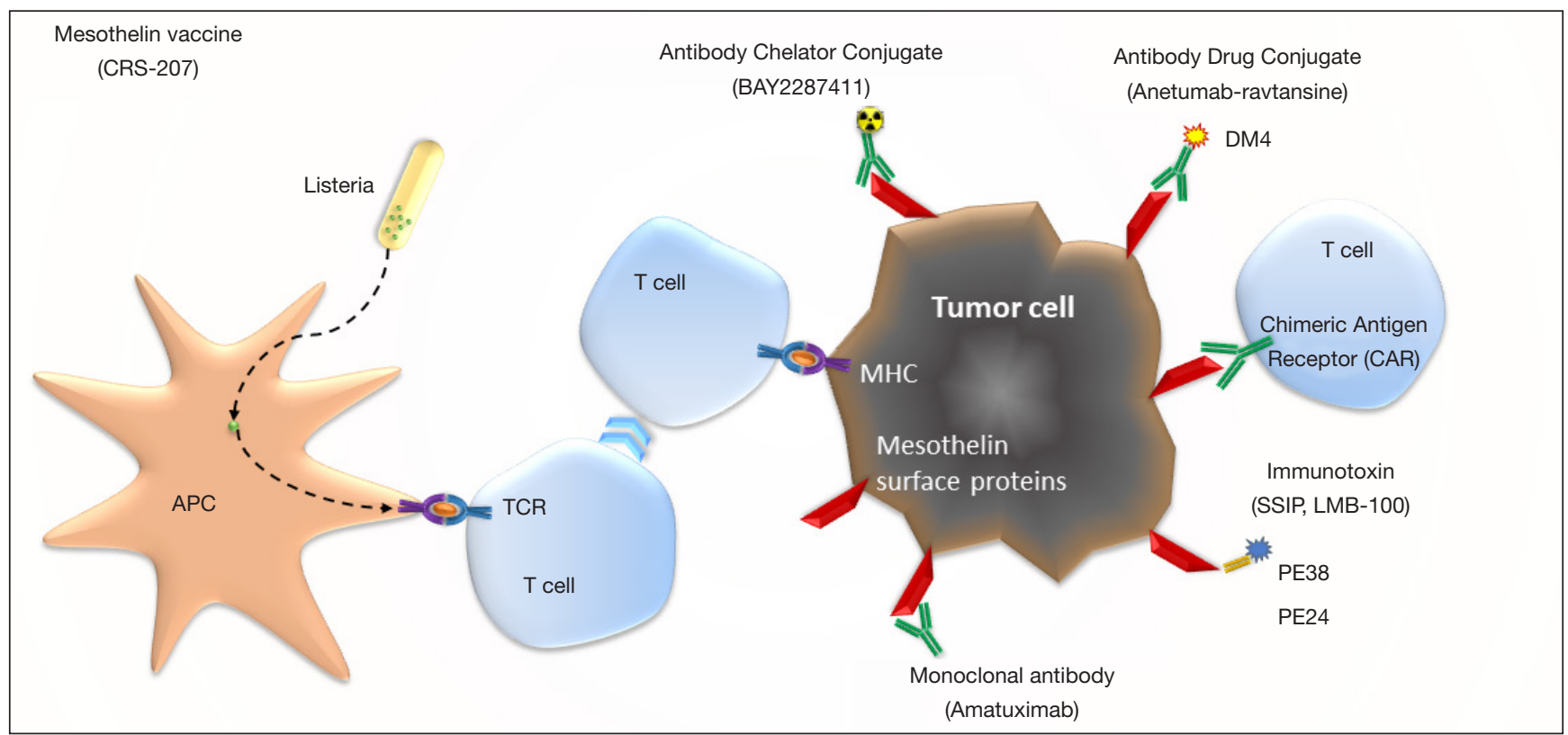

Figure 1 Therapeutics to target mesothelin. APC, antigen presenting cell; MHC, major histocompatibility complex; PE, pseudomonas exotoxin; TCR, T cell receptor. Different mechanisms of targeting mesothelin, a suface glycoprotein.

The third approach is via antibody drug conjugates. Anetumab ravtansine, is an anti-mesothelin antibody fused to DM4, a maytansinoid tubulin inhibitor. After internalization it releases the DM4 metabolite in the tumor cell. In a phase II trial, presented at WCLC 2017, anetumab ravtansine had an objective response of $8.4 \%$ and was not superior to vinorelbine with respect to PFS (41). A study randomizing between anetumab ravtansine plus pembrolizumab versus pembrolizumab alone is now recruiting (NCT03126630).

BAY2287411 is a thorium-227-labeled antibody-chelator conjugate, currently being tested in a phase I clinical trial (NCT03507452).

Cancer vaccines are designed to induce a tumor-specific immune response. CRS-207 uses a live-attenuated Listeria monocytogenes strain engineered to express mesothelin. It has been tested in phase I trials, as single agent or in combination with chemotherapy, but although it induces a change in tumor micro-environment and seems to give small benefit, it is no longer tested anymore $(42,43)$. Another cancer vaccine (JNJ-64041757) is also no longer in development.

Over the last two decades many different trials have been performed, unfortunately most without clear effect.

Anti-mesothelin chimeric antigen receptor (CAR) T cells are modified from autologous patient $\mathrm{T}$ cells, to express a mesothelin-binding T-cell receptor, and providing antigen specificity to T-cells against tumor associated antigens on the cell surface. Mesothelin CARs are being tested in several trials. A recent phase I basket trial with CAR-Tcells engineered by lentiviral transduction showed it was well tolerated, but showed limited clinical benefit (44). Inefficient $\mathrm{T}$ cell infiltration and short persistence by systemic delivery are common obstacles for solid tumor CAR-T cell therapy. Other problems are a cytokine release syndrome and neurotoxicity. Different phase I clinical trials are ongoing (NCT03054298, NCT02414269, NCT03638206).

\section{Arginine deprivation}

For the subgroup of sarcomatoid mesothelioma only recently the importance of the arginine succinate synthase (ASS) pathway has been identified $(45,46)$. Using a drug to deplete the body from circulating arginine, the sarcomatoid cells will die due to their inability to endogenously produce arginine. Somewhere during the development of the malignant expression of these cells, there has occurred a loss of the ASS enzyme. In a randomized phase II trial arginine deprivation with ADI-PEG20 improved PFS, but not OS, 
over BSC, in patients with ASS1 deficient mesothelioma (47). A phase II/III trial randomizes 386 patients with mixedtype and sarcomatoid mesothelioma, to platinum plus pemetrexed, and either ADI-PEG20 or placebo and is currently recruiting patients (NCT02709512).

\section{Surgery in MPM}

The role of surgery in diagnosis and palliation has been well established. In the curative setting, surgery has been performed in patients with MPM for several decades as part of a multi-modality setting. Its primary goal is to eradicate all visible tumor and to allow other modalities to kill the remaining microscopic disease. Different approaches have been investigated, with an Extra-Pleural Pneumonectomy (EPP) being the most radical approach. Several nonrandomized phase II studies showed promising outcomes in highly selected patient groups $(48,49)$. A small but randomized study in the UK (MARS) indicated that toxicity and morbidity was considerable and did not show any signs of improvement (50). The study execution, however, was criticized but gave rise to renewed interest in more limited resections: pleurectomy decortication (P/D). Different ways of performing this resection of all visible tumor with leaving the lung intact have been published. The major problem which is currently under investigation is how the different PD interventions can be compared. In the UK, the MARS2 study investigates the impact of extended pleurectomy/decortication (eP/D) when added to chemotherapy alone (51). The EORTC 1205 study tests the sequence of chemotherapy and eP/D in a randomized study in 64 patients (52). Multimodality treatment is recommended only within clinical trials.

\section{Selection of the best therapy for a patient}

In the last years many promising studies with systemic agents have been reported but it all comes down to a longterm benefit in only $20-25 \%$ of patients. Despite many investigations, we have not been able to find reliable biomarkers to select for any of the new therapies. It is therefore generally accepted that a platinum with antifolate combination, potentially including bevacizumab, remains the cornerstone of first-line treatment until a new randomized study beats this standard.

As general recommendation, patients can be selected using the EORTC or CALGB prognostic models for certain (combination) surgical approaches (53) until better biomarkers have been identified.

In further lines no standard therapy is available. Possibilities include chemotherapy (pemetrexed retreatment, gemcitabine or vinorelbine) or immune checkpoint inhibitors (PD-1 +/- CTLA-4). Since ICI have a higher ORR and less toxicity than chemotherapy, possibly this is preferred when available. In the next years several trials with combining agents will be published.

The high expression of mesothelin in epithelioid mesothelioma provides a promising way for use of targeted therapy, but there are still some obstacles to overcome.

We need to continue to encourage patients to enroll in studies to identify which combination of modalities is the most promising and has the least toxicity. It is strongly recommended that these clinical investigations all have strong translational programs.

\section{Acknowledgments}

Funding: None.

\section{Footnote}

Conflicts of Interest: The authors have no conflicts of interest to declare.

Ethical Statement: The authors are accountable for all aspects of the work in ensuring that questions related to the accuracy or integrity of any part of the work are appropriately investigated and resolved.

Open Access Statement: This is an Open Access article distributed in accordance with the Creative Commons Attribution-NonCommercial-NoDerivs 4.0 International License (CC BY-NC-ND 4.0), which permits the noncommercial replication and distribution of the article with the strict proviso that no changes or edits are made and the original work is properly cited (including links to both the formal publication through the relevant DOI and the license). See: https://creativecommons.org/licenses/by-ncnd/4.0/.

\section{References}

1. Muers MF, Stephens RJ, Fisher P, et al. Active symptom control with or without chemotherapy in the treatment of 
patients with malignant pleural mesothelioma (MS01): a multicentre randomised trial. Lancet 2008;371:1685-94.

2. Vogelzang NJ, Rusthoven JJ, Symanowski J, et al. Phase III study of pemetrexed in combination with cisplatin versus cisplatin alone in patients with malignant pleural mesothelioma. J Clin Oncol 2003;21:2636-44.

3. Zucali PA, Giovannetti E, Destro A, et al. Thymidylate synthase and excision repair cross-complementing group-1 as predictors of responsiveness in mesothelioma patients treated with pemetrexed/carboplatin. Clin Cancer Res 2011;17:2581-90.

4. Ohta Y, Shridhar V, Bright RK, et al. VEGF and VEGF type $\mathrm{C}$ play an important role in angiogenesis and lymphangiogenesis in human malignant mesothelioma tumours. Br J Cancer 1999;81:54-61.

5. Langerak AW, De Laat PA, Van Der Linden-Van Beurden CA, et al. Expression of platelet-derived growth factor (PDGF) and PDGF receptors in human malignant mesothelioma in vitro and in vivo. $\mathrm{J}$ Pathol 1996;178:151-60.

6. Kumar-Singh S, Weyler J, Martin MJ, et al. Angiogenic cytokines in mesothelioma: a study of VEGF, FGF-1 and -2, and TGF beta expression. J Pathol 1999;189:72-8.

7. Strizzi L, Catalano A, Vianale G, et al. Vascular endothelial growth factor is an autocrine growth factor in human malignant mesothelioma. J Pathol 2001;193:468-75.

8. Cacciotti P, Strizzi L, Vianale G, et al. The presence of simian-virus 40 sequences in mesothelioma and mesothelial cells is associated with high levels of vascular endothelial growth factor. Am J Respir Cell Mol Biol 2002;26:189-93.

9. Buikhuisen WA, Scharpfenecker M, Griffioen AW, et al. A Randomized Phase II Study Adding Axitinib to Pemetrexed-Cisplatin in Patients with Malignant Pleural Mesothelioma: A Single-Center Trial Combining Clinical and Translational Outcomes. J Thorac Oncol 2016;11:758-68.

10. Zalcman G, Mazieres J, Margery J, et al. Bevacizumab for newly diagnosed pleural mesothelioma in the Mesothelioma Avastin Cisplatin Pemetrexed Study (MAPS): a randomised, controlled, open-label, phase 3 trial. Lancet 2016;387:1405-14.

11. Kindler HL, Karrison TG, Gandara DR, et al. Multicenter, double-blind, placebo-controlled, randomized phase II trial of gemcitabine/cisplatin plus bevacizumab or placebo in patients with malignant mesothelioma. J Clin Oncol 2012;30:2509-15.

12. Dowell JE, Dunphy FR, Taub RN, et al. A multicenter phase II study of cisplatin, pemetrexed, and bevacizumab in patients with advanced malignant mesothelioma. Lung Cancer 2012;77:567-71.

13. Garland LL, Chansky K, Wozniak AJ, et al. Phase II study of cediranib in patients with malignant pleural mesothelioma: SWOG S0509. J Thorac Oncol 2011;6:1938-45.

14. Campbell NP, Kunnavakkam R, Leighl N, et al. Cediranib in patients with malignant mesothelioma: a phase II trial of the University of Chicago Phase II Consortium. Lung Cancer 2012;78:76-80.

15. Laurie SA, Hao D, Leighl NB, et al. A phase II trial of dovitinib in previously-treated advanced pleural mesothelioma: The Ontario Clinical Oncology Group. Lung Cancer 2017;104:65-9.

16. Scagliotti GV, Gaafar R, Nowak AK, et al. Nintedanib in combination with pemetrexed and cisplatin for chemotherapy-naive patients with advanced malignant pleural mesothelioma (LUME-Meso): a double-blind, randomised, placebo-controlled phase 3 trial. Lancet Respir Med 2019;7:569-80.

17. Dubey S, Janne PA, Krug L, et al. A phase II study of sorafenib in malignant mesothelioma: results of Cancer and Leukemia Group B 30307. J Thorac Oncol 2010;5:1655-61.

18. Papa S, Popat S, Shah R, et al. Phase 2 study of sorafenib in malignant mesothelioma previously treated with platinum-containing chemotherapy. J Thorac Oncol 2013;8:783-7.

19. Laurie SA, Gupta A, Chu Q, et al. Brief report: a phase II study of sunitinib in malignant pleural mesothelioma. the NCIC Clinical Trials Group. J Thorac Oncol 2011;6:1950-4.

20. Nowak AK, Millward MJ, Creaney J, et al. A phase II study of intermittent sunitinib malate as second-line therapy in progressive malignant pleural mesothelioma. J Thorac Oncol 2012;7:1449-56.

21. Buikhuisen WA, Burgers JA, Vincent AD, et al. Thalidomide versus active supportive care for maintenance in patients with malignant mesothelioma after first-line chemotherapy (NVALT 5): an open-label, multicentre, randomised phase 3 study. Lancet Oncol 2013;14:543-51.

22. Jahan T, Gu L, Kratzke R, et al. Vatalanib in malignant mesothelioma: a phase II trial by the Cancer and Leukemia Group B (CALGB 30107). Lung Cancer 2012;76:393-6.

23. Dudek AZ, Wang XF, Gu L, et al. Randomized phase 2 study of maintenance pemetrexed (Pem) versus observation (Obs) for patients (pts) with malignant pleural mesothelioma (MPM) without progression after first-line 
chemotherapy: Cancer and Leukemia Group B (CALGB) 30901 (Alliance). J Clin Oncol 2019;37:8517.

24. Burgers SA, de Gooijer C, Cornelissen R, et al. LBA92Switch maintenance gemcitabine after first-line chemotherapy in patients with malignant mesothelioma: A multicenter open label phase II trial (NVALT19). Ann Oncol 2019;30. doi: 10.1093/annonc/mdz394.092.

25. Krug LM, Kindler HL, Calvert H, et al. Vorinostat in patients with advanced malignant pleural mesothelioma who have progressed on previous chemotherapy (VANTAGE-014): a phase 3, double-blind, randomised, placebo-controlled trial. Lancet Oncol 2015;16:447-56.

26. McLoughlin KC, Kaufman AS, Schrump DS. Targeting the epigenome in malignant pleural mesothelioma. Transl Lung Cancer Res 2017;6:350-65.

27. LaFave LM, Beguelin W, Koche R, et al. Loss of BAP1 function leads to EZH2-dependent transformation. Nat Med 2015;21:1344-9.

28. Alley EW, Lopez J, Santoro A, et al. Clinical safety and activity of pembrolizumab in patients with malignant pleural mesothelioma (KEYNOTE-028): preliminary results from a non-randomised, open-label, phase $1 \mathrm{~b}$ trial. Lancet Oncol 2017;18:623-30.

29. Metaxas Y, Rivalland G, Mauti LA, et al. Pembrolizumab as Palliative Immunotherapy in Malignant Pleural Mesothelioma. J Thorac Oncol 2018;13:1784-91.

30. Popat S, Curioni-Fontecedro A, Polydoropoulou V, et al. LBA91_PRA multicentre randomized phase III trial comparing pembrolizumab $(\mathrm{P})$ vs single agent chemotherapy (CT) for advanced pre-treated malignant pleural mesothelioma (MPM): Results from the European Thoracic Oncology Platform (ETOP 9-15) PROMISEmeso trial. Ann Oncol 2019;30. doi: 10.1093/annonc/ mdz394.091

31. Quispel-Janssen J, van der Noort V, de Vries JF, et al. Programmed Death 1 Blockade With Nivolumab in Patients With Recurrent Malignant Pleural Mesothelioma. J Thorac Oncol 2018;13:1569-76.

32. Okada M, Kijima T, Aoe K, et al. Clinical Efficacy and Safety of Nivolumab: Results of a Multicenter, Openlabel, Single-arm, Japanese Phase II study in Malignant Pleural Mesothelioma (MERIT). Clin Cancer Res 2019;25:5485-92.

33. Scherpereel A, Mazieres J, Greillier L, et al. Nivolumab or nivolumab plus ipilimumab in patients with relapsed malignant pleural mesothelioma (IFCT-1501 MAPS2): a multicentre, open-label, randomised, non-comparative, phase 2 trial. Lancet Oncol 2019;20:239-53.
34. Hassan R, Thomas A, Nemunaitis JJ, et al. Efficacy and Safety of Avelumab Treatment in Patients With Advanced Unresectable Mesothelioma: Phase 1b Results From the JAVELIN Solid Tumor Trial. JAMA Oncol 2019;5:351-7.

35. Maio M, Scherpereel A, Calabro L, et al. Tremelimumab as second-line or third-line treatment in relapsed malignant mesothelioma (DETERMINE): a multicentre, international, randomised, double-blind, placebocontrolled phase 2b trial. Lancet Oncol 2017;18:1261-73.

36. Disselhorst MJ, Quispel-Janssen J, Lalezari F, et al. Ipilimumab and nivolumab in the treatment of recurrent malignant pleural mesothelioma (INITIATE): results of a prospective, single-arm, phase 2 trial. Lancet Respir Med 2019;7:260-70

37. Calabrò L, Morra A, Giannarelli D, et al. Tremelimumab combined with durvalumab in patients with mesothelioma (NIBIT-MESO-1): an open-label, non-randomised, phase 2 study. Lancet Respir Med 2018;6:451-60.

38. Aerts JG, de Goeje PL, Cornelissen R, et al. Autologous Dendritic Cells Pulsed with Allogeneic Tumor Cell Lysate in Mesothelioma: From Mouse to Human. Clin Cancer Res 2018;24:766-76.

39. Hassan R, Kindler HL, Jahan T, et al. Phase II clinical trial of amatuximab, a chimeric antimesothelin antibody with pemetrexed and cisplatin in advanced unresectable pleural mesothelioma. Clin Cancer Res 2014;20:5927-36.

40. Hassan R, Sharon E, Thomas A, et al. Phase 1 study of the antimesothelin immunotoxin SS1P in combination with pemetrexed and cisplatin for front-line therapy of pleural mesothelioma and correlation of tumor response with serum mesothelin, megakaryocyte potentiating factor, and cancer antigen 125. Cancer 2014;120:3311-9.

41. Kindler HL, Novello S, Fennell D, et al. OA 02.01 Randomized Phase II Study of Anetumab Ravtansine or Vinorelbine in Patients with Metastatic Pleural Mesothelioma. J Thorac Oncol 2017;12:S1746.

42. Hassan R, Alley E, Kindler H, et al. Clinical Response of Live-Attenuated, Listeria monocytogenes Expressing Mesothelin (CRS-207) with Chemotherapy in Patients with Malignant Pleural Mesothelioma. Clin Cancer Res 2019;25:5787-98.

43. Le DT, Brockstedt DG, Nir-Paz R, et al. A live-attenuated Listeria vaccine (ANZ-100) and a live-attenuated Listeria vaccine expressing mesothelin (CRS-207) for advanced cancers: phase I studies of safety and immune induction. Clin Cancer Res 2012;18:858-68.

44. Haas AR, Tanyi JL, O'Hara MH, et al. Phase I Study of Lentiviral-Transduced Chimeric Antigen Receptor- 
Modified T Cells Recognizing Mesothelin in Advanced Solid Cancers. Mol Ther 2019;27:1919-29.

45. Szlosarek PW, Klabatsa A, Pallaska A, et al. In vivo loss of expression of argininosuccinate synthetase in malignant pleural mesothelioma is a biomarker for susceptibility to arginine depletion. Clin Cancer Res 2006;12:7126-31.

46. Delage B, Fennell DA, Nicholson L, et al. Arginine deprivation and argininosuccinate synthetase expression in the treatment of cancer. Int J Cancer 2010;126:2762-72.

47. Szlosarek PW, Steele JP, Nolan L, et al. Arginine Deprivation With Pegylated Arginine Deiminase in Patients With Argininosuccinate Synthetase 1-Deficient Malignant Pleural Mesothelioma: A Randomized Clinical Trial. JAMA Oncol 2017;3:58-66.

48. Lauk O, Hoda MA, de Perrot M, et al. Extrapleural pneumonectomy after induction chemotherapy: perioperative outcome in 251 mesothelioma patients from three high-volume institutions. Ann Thorac Surg 2014;98:1748-54.

49. Cao CQ, Yan TD, Bannon PG, et al. A systematic review of extrapleural pneumonectomy for malignant pleural

Cite this article as: Disselhorst MJ, Baas P. Chemotherapy options versus "novel" therapies: how should we treat patients with malignant pleural mesothelioma. Transl Lung Cancer Res 2020;9(Suppl 1):S77-S85. doi: 10.21037/tlcr.2020.01.16 mesothelioma. J Thorac Oncol 2010;5:1692-703.

50. Treasure T, Lang-Lazdunski L, Waller D, et al. Extrapleural pneumonectomy versus no extra-pleural pneumonectomy for patients with malignant pleural mesothelioma: clinical outcomes of the Mesothelioma and Radical Surgery (MARS) randomised feasibility study. Lancet Oncol 2011;12:763-72.

51. Trialists M, Lim E. OA 02.07 Surgical Selection in Pleurectomy Decortication for Mesothelioma \&\#x2013; an Overview from Screening and Selection from MARS 2 Pilot. J Thorac Oncol 2017;12:S1748.

52. Raskin J, Surmont V, Cornelissen R, et al. A randomized phase II study of pleurectomy/decortication preceded or followed by (neo-)adjuvant chemotherapy in patients with early stage malignant pleural mesothelioma (EORTC 1205). Transl Lung Cancer Res 2018;7:593-8.

53. Sandri A, Guerrera F, Roffinella M, et al. Validation of EORTC and CALGB prognostic models in surgical patients submitted to diagnostic, palliative or curative surgery for malignant pleural mesothelioma. J Thorac Dis 2016;8:2121-7. 